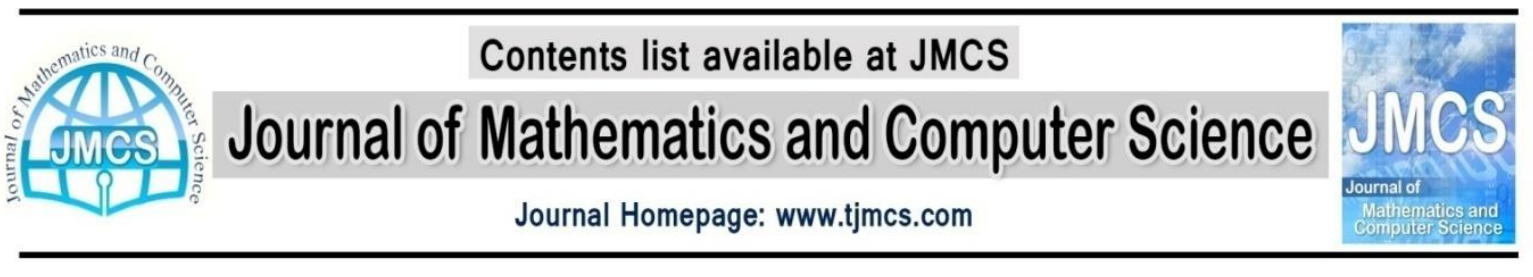

\title{
Linear Equations and Systems in Fuzzy Environment
}

\author{
Sanhita Banerjee ${ }^{1, *}$, Tapan Kumar Roy ${ }^{1,+}$ \\ ${ }^{1}$ Department of Mathematics, Indian Institute of Engineering Science and Technology, Shibpur, \\ (formerly BESU, Shibpur) Howrah-711103, West Bengal, India \\ *sanhita.banerjee88@gmail.com \\ +roy_t_k@yahoo.co.in
}

Article history:

Received December 2014

Accepted January 2015

Available online January 2015

\section{Abstract}

The paper discusses fuzzy real and complex linear equations and system of linear equations with coefficients as crisp and the right-hand side as generalized trapezoidal fuzzy number where fuzzy numbers have been represented with mean and semi width. We have solved each case by using the concept of Strong and Weak solution with numerical examples.

Keywords: Fuzzy Linear Equation, Fuzzy System of Linear Equations, Generalized Trapezoidal Fuzzy Number (GTrFN), Strong and Weak solutions.

\section{Introduction}

Systems of linear equations are mainly used to solve several problems in various areas of mathematical, physical and engineering sciences such as circuit analysis, structural mechanics, heat transport, fluid flow etc. In most of the applications, the system's parameters and measurements are vague or imprecise. In that situation we can represent them with fuzzy numbers rather than crisp numbers.

In literature, standard analytical techniques to solve fuzzy linear equation ware proposed by Buckley and $\mathrm{Qu}[5,6,8,9]$. Buckley [6,7] considered the solution of linear fuzzy equations using Classical methods, Zadeh's extension principle and the concept of fuzzy numbers and arithmetic operations on it introduced by Zadeh [10,11]. Fuzzy linear systems are the linear systems whose parameters are all or partially represented by fuzzy numbers. A general model for solving a Fuzzy linear system whose coefficient matrix is crisp and the right-hand side column is an arbitrary fuzzy number was first proposed by Friedman et al. [12]. They have used the parametric form of fuzzy numbers and replace

the original $n \times n$ fuzzy system by a $2 n \times 2 n$ crisp system. Fuzzy linear system has been studied by several authors $[1,2,4,13,14,15,16,17,18]$.

In this paper we have used another approach to solve real and complex fuzzy linear equations and system of linear equations where fuzzy numbers have been represented with mean and semi width. In Section-3 we have solved real and complex fuzzy linear equations and system of linear equations respectively by using the concept of Strong and Weak solution and coefficients are taken as 
Generalized Trapezoidal Fuzzy Numbers (GTrFNs). We have also illustrated each case by numerical examples along the methods.

\section{Preliminaries}

Definition-2.1: The Signum function of a real number $x$ is defined as follows:

$$
\operatorname{sgn}(x):= \begin{cases}-1 & \text { if } x<0 \\ 0 & \text { if } x=0 \\ 1 & \text { if } x>0\end{cases}
$$

Definition-2.2: Interval Number: An interval number is a closed and bounded set of real numbers.

$$
A=[a, b]=\{x: a \leq x \leq b \forall a, b, x \in R\}
$$

Any interval of real number can be expressed as an interval number.

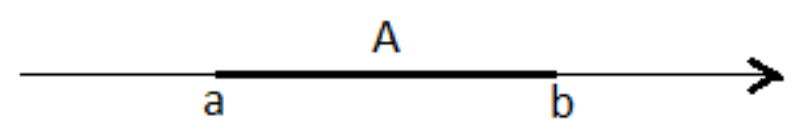

\section{Fig2.1: Interval number A}

Any Interval number may be represented with its mean and semi width.

An interval number $\mathrm{A}(=[a, b])$ may be denoted as $\langle m(A), w(A)\rangle$ where $m(A)=\frac{a+b}{2}$ and $w(A)=$ $\frac{b-a}{2}$

\section{Definition-2.3: Interval Arithmetic:}

For any two interval numbers $A=\langle m(A), w(A)\rangle, B=\langle m(B), w(B)\rangle$ and scalar k, we have

1. $A=B$ iff $m(A)=m(B)$ and $w(A)=w(B)$

2. $A+B=\langle m(A)+m(B), w(A)+w(B)\rangle$

3. $A-B=\langle m(A)-m(B), w(A)+w(B)\rangle$

4. $k A=\left\{\begin{array}{c}\langle k m(A), k w(A)\rangle, k \geq 0 \\ \langle k m(A),-k w(A)\rangle, k<0\end{array}\right.$

5. $A^{-1}=\langle m(A), w(A)\rangle^{-1}=\left\langle\frac{m(A)}{\{m(A)\}^{2}-\{w(A)\}^{2}}, \frac{w(A)}{\{m(A)\}^{2}-\{w(A)\}^{2}}\right\rangle$

Definition-2.4: A fuzzy number $\tilde{X}$ in parametric form is defined as $m_{\tilde{X}}(\propto)+w_{\tilde{X}}(\propto) K$ where $m_{\tilde{X}}(\propto)$ is the mean of $X, w_{\tilde{X}}(\propto)$ is the semi width of $X$ and $K=[-1,1]$ is the constant interval.

A fuzzy number in parametric form is completely determined by a pair $\left\langle m_{\tilde{X}}(\propto), w_{\tilde{X}}(\propto)\right\rangle$ of functions $m_{\tilde{X}}(\propto), w_{\tilde{X}}(\propto), 0 \leq \propto \leq 1$, which satisfy the following requirements:

1. $\left|\frac{d m_{\widetilde{X}}(\propto)}{d \propto}\right|>\frac{d w_{\widetilde{X}}(\propto)}{d \propto}$

2. $w_{\tilde{X}}(\propto) \geq 0 \quad \forall \propto \in(0,1]$

\section{Definition 2.5: Fuzzy linear equation (FLE):}

These are the equations in which coefficients and unknowns are fuzzy numbers, and formulas are constructed by operations of fuzzy arithmetic. These equations are of three very simple types: $\tilde{A} \tilde{X}=$ 
$\tilde{B}, \tilde{X}+\tilde{A}=\tilde{B}$ and $\tilde{A} \tilde{X}+\tilde{B}=\tilde{C}$ where $\tilde{A}, \tilde{B}, \tilde{C}$ are fuzzy numbers, and $\tilde{X}$ is an unknown fuzzy number for which either of the equations is to be satisfied.

Definition-2.6: The $n \times n$ linear system

$a_{11} x_{1}+a_{12} x_{2}+\cdots+a_{1 n} x_{n}=b_{1}$
$a_{21} x_{1}+a_{22} x_{2}+\cdots+a_{2 n} x_{n}=b_{2}$
$\vdots$
$a_{n 1} x_{1}+a_{n 2} x_{2}+\cdots+a_{n n} x_{n}=b_{n}$

where the co-efficient matrix $A=\left(a_{i j}\right), 1 \leq i \leq \mathrm{n}, 1 \leq \mathrm{j} \leq \mathrm{n}$ is a crisp real $n \times n$ matrix and $b_{i}, 1 \leq i \leq n$ are fuzzy numbers is called a fuzzy linear system.

Theorem-2.1: A fuzzy number vector $\left(x_{1}, x_{2}, \ldots, x_{n}\right)^{T}$ given by

$x_{i}=\left\langle m\left(x_{i}\right), w\left(x_{i}\right)\right\rangle, 1 \leq i \leq n$, is called a solution of the fuzzy linear system (2.1) if

$\sum_{j=1}^{n} a_{i j} m\left(x_{i}\right)=m\left(b_{i}\right), \sum_{j=1}^{n} a_{i j} w\left(x_{i}\right) \operatorname{sgn}\left(a_{i j}\right)=w\left(b_{i}\right)$

Definition-2.7: If $x=\left(m\left(x_{1}\right), m\left(x_{2}\right), \ldots, m\left(x_{n}\right), w\left(x_{1}\right), w\left(x_{2}\right), \ldots, w\left(x_{n}\right)\right)^{T}$ is a solution of (2.1) and for each $1 \leq i \leq n, w\left(x_{i}\right) \geq 0$ hold, then the solution $\mathrm{x}$ is called a strong solution of the system (2.1) and in this case the strong solution is $x_{i}=\left\langle m\left(x_{i}\right), w\left(x_{i}\right)\right\rangle, 1 \leq i \leq n$.

Definition-2.8: If $x=\left(m\left(x_{1}\right), m\left(x_{2}\right), \ldots, m\left(x_{n}\right), w\left(x_{1}\right), w\left(x_{2}\right), \ldots, w\left(x_{n}\right)\right)^{T}$ is a solution of (2.1) and for some $\in[1, n], w\left(x_{i}\right)<0$ hold, then the solution $\mathrm{x}$ is called a weak solution of the system (2.1) and in this case the weak solution is $x_{i}=\left\langle m\left(x_{i}\right),-w\left(x_{i}\right)\right\rangle, 1 \leq i \leq n$.

\section{Solution procedures}

\subsection{Solution procedure for real fuzzy linear equation}

Consider the equation $A \tilde{X}+\tilde{B}=\tilde{C}$

Where $\mathrm{A}$ is a crisp number, $\mathrm{B}$ and $\mathrm{C}$ are known fuzzy numbers and $\mathrm{X}$ is unknown fuzzy number.

$\tilde{B}=\langle m(B), w(B)\rangle$ and $\tilde{C}=\langle m(C), w(C)\rangle$

Let $\tilde{X}=\langle m(X), w(X)\rangle$

Then equation (3.1) becomes

$\mathrm{A}\{m(X)+K w(X)\}+\{m(B)+K w(B)\}=\{m(C)+K w(C)\}$

or, $A \cdot m(X)+m(B)=m(C) \Rightarrow m(X)=\frac{m(C)-m(B)}{A}$

and $A \cdot \operatorname{sgn}(A) \cdot w(X)+w(B)=w(C) \Rightarrow w(X)=\frac{w(C)-w(B)}{A \cdot \operatorname{sgn}(A)}$

Example3.1: Consider the equation $A \tilde{X}+\tilde{B}=\tilde{C}$

Where $A=9, \tilde{B}=(-4,-3,-2,-1 ; 0.8), \tilde{C}=(3,5,7,9 ; 0.9)$

$\tilde{B}=\left\langle-2.5,1.5-\frac{\alpha}{\omega}\right\rangle$ and $\tilde{C}=\left\langle 6,3-\frac{2 \propto}{\omega}\right\rangle$

where $\omega=\min (0.8,0.9)=0.8$

Let $\tilde{X}=\langle m(X), w(X)\rangle$

Then the given equation becomes

9. $m(X)-2.5=6 \Rightarrow m(X)=\frac{8.5}{9}$ 
and $9 . w(X)+1.5-\frac{\alpha}{\omega}=3-\frac{2 \alpha}{\omega} \Rightarrow w(X)=\frac{1}{9}\left(1.5-\frac{\alpha}{\omega}\right)$

Here $w(X)>0$, so the solution is a strong solution and the solution is

$\tilde{X} \approx\left(\frac{6}{9}, \frac{7}{9}, \frac{9}{9}, \frac{10}{9} ; 0.8\right)$

\subsection{Solution procedure for complex fuzzy linear equations}

Consider the equation $A \tilde{Z}+\tilde{B}=\tilde{C}$

Where $\mathrm{A}, \mathrm{B}$ and $\mathrm{C}$ are known complex fuzzy numbers and $\mathrm{X}$ is unknown complex fuzzy number.

$A=a_{1}+i a_{2}, \tilde{B}=\widetilde{b_{1}}+i \widetilde{b_{2}}, \tilde{C}=\widetilde{c_{1}}+i \widetilde{c_{2}}$,

$\tilde{B}=\langle m(B), w(B)\rangle=\left\langle m\left(b_{1}\right)+i m\left(b_{2}\right), w\left(b_{1}\right)+i w\left(b_{2}\right)\right\rangle$

and $\tilde{C}=\langle m(C), w(C)\rangle=\left\langle m\left(c_{1}\right)+i m\left(c_{2}\right), w\left(c_{1}\right)+i w\left(c_{2}\right)\right\rangle$

Let $\tilde{Z}=\widetilde{x_{1}}+i \widetilde{x_{2}}$

We denote $\tilde{Z}$ as $\langle m(Z), w(Z)\rangle=\left\langle m\left(x_{1}\right)+i m\left(x_{2}\right), w\left(x_{1}\right)+i w\left(x_{2}\right)\right\rangle$.

Then equation (3.2) becomes

$\left(a_{1}+i a_{2}\right)\{m(X)+K w(X)\}+\{m(B)+K w(B)\}=\{m(C)+K w(C)\}$

or, $\left(a_{1}+i a_{2}\right) \cdot m(X)+m(B)=m(C)$

$\Rightarrow m(X)=\frac{m(C)-m(B)}{\left(a_{1}+i a_{2}\right)}$

and $\left(a_{1} \cdot \operatorname{sgn}\left(a_{1}\right)+i a_{2} \cdot \operatorname{sgn}\left(a_{2}\right)\right) w(A)+w(B)=w(C)$

$\Rightarrow w(X)=\frac{w(C)-w(B)}{\left(a_{1} \cdot \operatorname{sgn}\left(a_{1}\right)+i a_{2} \cdot \operatorname{sgn}\left(a_{2}\right)\right)}$

Example3.2: Consider the equation $A \tilde{Z}+\tilde{B}=\tilde{C}$

Where $A=10-7 i, \widetilde{B}=(-8,-7,-6,-5 ; 0.8)+i(1,2,3,4 ; 0.9)$,

$\tilde{C}=(3,5,7,9 ; 0.9)+i(12,14,16,18 ; 0.8)$

$\tilde{B}=\left\langle-6.5,1.5-\frac{\alpha}{\omega}\right\rangle+i\left\langle 2.5,1.5-\frac{\alpha}{\omega}\right\rangle$ and $\tilde{C}=\left\langle 6,3-\frac{2 \alpha}{\omega}\right\rangle+i\left\langle 15,3-\frac{2 \alpha}{\omega}\right\rangle$

where $\omega=\min (0.8,0.9)=0.8$

Let $\tilde{Z}=\tilde{X}+i \tilde{Y} \approx\langle m(Z), w(Z)\rangle=\langle m(X)+i m(Y), w(X)+i w(Y)\rangle$

Then the given equation becomes

$(10-7 i) \cdot m(X)-6.5+2.5 i=6+15 i \Rightarrow m(X)=\frac{12.5+12.5 i}{10-7 i}$

and $(10+7 i) \cdot w(X)+\left(1.5-\frac{\alpha}{\omega}\right)+i\left(1.5-\frac{\alpha}{\omega}\right)=\left(3-\frac{2 \alpha}{\omega}\right)+i\left(3-\frac{2 \alpha}{\omega}\right)$

$\Rightarrow w(X)=\frac{\left(1.5-\frac{\alpha}{\omega}\right)(1+i)}{(10+7 i)}$

Here $w(X)>0$, so the solution is a strong solution and the solution is

$\tilde{Z} \approx\left(\frac{12}{51}, \frac{29}{51}, \frac{46}{51}, \frac{63}{51} ; 0.8\right)+i\left(\frac{208}{51}, \frac{211}{51}, \frac{214}{51}, \frac{217}{51} ; 0.8\right)$

\subsection{Solution procedure for $\boldsymbol{n} \times \boldsymbol{n}$ real fuzzy linear system}

Consider the $n \times n$ linear system

$a_{11} x_{1}+a_{12} x_{2}+\cdots+a_{1 n} x_{n}=b_{1}$ 
$a_{21} x_{1}+a_{22} x_{2}+\cdots+a_{2 n} x_{n}=b_{2}$

$\vdots$

$a_{n 1} x_{1}+a_{n 2} x_{2}+\cdots+a_{n n} x_{n}=b_{n}$

where the co-efficient matrix $A=\left(a_{i j}\right), 1 \leq i \leq n, 1 \leq j \leq \mathrm{n}$, is a crisp real $n \times n$ matrix and $b_{i}, 1 \leq i \leq n$ are fuzzy numbers.

Let us now rearrange the linear system so that the unknowns are $m\left(x_{i}\right), w\left(x_{i}\right)$, $1 \leq i \leq n$, and the right hand side column is $b=\left(m\left(b_{1}\right)+K w\left(b_{1}\right), m\left(b_{2}\right)+K w\left(b_{2}\right), \ldots, m\left(b_{n}\right)+K w\left(b_{n}\right)\right)^{T}$.

We get the crisp linear system

$$
\begin{aligned}
& a_{11}\left\{m\left(x_{1}\right),+K w\left(x_{1}\right)\right\}+a_{12}\left\{m\left(x_{2}\right),+K w\left(x_{2}\right)\right\}+\cdots \\
& +a_{1 n}\left\{m\left(x_{n}\right),+K w\left(x_{n}\right)\right\}=\left\{m\left(b_{1}\right),+K w\left(b_{1}\right)\right\} \\
& \vdots \\
& a_{21}\left\{m\left(x_{1}\right),+K w\left(x_{1}\right)\right\}+a_{22}\left\{m\left(x_{2}\right),+K w\left(x_{2}\right)\right\}+\cdots \\
& +a_{2 n}\left\{m\left(x_{n}\right),+K w\left(x_{n}\right)\right\}=\left\{m\left(b_{2}\right),+K w\left(b_{2}\right)\right\} \\
& a_{n 1}\left\{m\left(x_{1}\right),+K w\left(x_{1}\right)\right\}+a_{n 2}\left\{m\left(x_{2}\right),+K w\left(x_{2}\right)\right\}+\cdots \\
& +a_{n n}\left\{m\left(x_{n}\right),+K w\left(x_{n}\right)\right\}=\left\{m\left(b_{n}\right),+K w\left(b_{n}\right)\right\} \\
&
\end{aligned}
$$

Comparing both sides we get two linear systems as

$$
\begin{aligned}
& a_{11} m\left(x_{1}\right)+a_{12} m\left(x_{2}\right)+\cdots+a_{1 n} m\left(x_{n}\right)=m\left(b_{1}\right) \\
& a_{21} m\left(x_{1}\right)+a_{22} m\left(x_{2}\right)+\cdots+a_{2 n} m\left(x_{n}\right)=m\left(b_{2}\right) \\
& \vdots \\
& a_{n 1} m\left(x_{1}\right)+a_{n 2} m\left(x_{2}\right)+\cdots+a_{n n} m\left(x_{n}\right)=m\left(b_{n}\right) \\
& \text { and } \\
& a_{11} w\left(x_{1}\right) \operatorname{sgn}\left(a_{11}\right)+a_{12} w\left(x_{2}\right) \operatorname{sgn}\left(a_{12}\right)+\cdots+a_{1 n} w\left(x_{n}\right) \operatorname{sgn}\left(a_{1 n}\right)=w\left(b_{1}\right) \\
& a_{21} w\left(x_{1}\right) \operatorname{sgn}\left(a_{21}\right)+a_{22} w\left(x_{2}\right) \operatorname{sgn}\left(a_{22}\right)+\cdots+a_{2 n} w\left(x_{n}\right) \operatorname{sgn}\left(a_{2 n}\right)=w\left(b_{2}\right) \\
& \vdots \\
& a_{n 1} w\left(x_{1}\right) \operatorname{sgn}\left(a_{n 1}\right)+a_{n 2} w\left(x_{2}\right) \operatorname{sgn}\left(a_{n 1}\right)+\cdots+a_{n n} w\left(x_{n}\right) \operatorname{sgn}\left(a_{n n}\right)=w\left(b_{n}\right)
\end{aligned}
$$

Using matrix notation we get $S x=b$

where $S=\left(\begin{array}{cc}S_{1} & 0 \\ 0 & S_{2}\end{array}\right)$ is $2 n \times 2 n$ matrix,

$S_{1}=\left(\begin{array}{cccc}a_{11} & a_{12} & \ldots & a_{1 n} \\ a_{21} & a_{22} & \ldots & a_{2 n} \\ \vdots & \vdots & \vdots & \vdots \\ a_{n 1} & a_{n 2} & \ldots & a_{n n}\end{array}\right)$ and $S_{2}=\left(\begin{array}{cccc}a_{11} \operatorname{sgn}\left(a_{11}\right) & a_{12} \operatorname{sgn}\left(a_{12}\right) & \ldots & a_{1 n} \operatorname{sgn}\left(a_{1 n}\right) \\ a_{21} \operatorname{sgn}\left(a_{21}\right) & a_{22} \operatorname{sgn}\left(a_{22}\right) & \ldots & a_{2 n} \operatorname{sgn}\left(a_{2 n}\right) \\ \vdots & \vdots & \vdots & \vdots \\ a_{n 1} \operatorname{sgn}\left(a_{n 1}\right) & a_{n 2} \operatorname{sgn}\left(a_{n 2}\right) & \ldots & a_{n n} \operatorname{sgn}\left(a_{n n}\right)\end{array}\right)$

$x=(m(x) w(x))^{T}$ where $m(x)=\left(m\left(x_{1}\right), m\left(x_{2}\right), \ldots, m\left(x_{n}\right)\right)^{T}$,

$w(x)=\left(w\left(x_{1}\right), w\left(x_{2}\right), \ldots, w\left(x_{n}\right)\right)^{T}$

and $b=(m(b) w(b))^{T}$ where $m(b)=\left(m\left(b_{1}\right), m\left(b_{2}\right), \ldots, m\left(b_{n}\right)\right)^{T}$,

$w(b)=\left(w\left(b_{1}\right), w\left(b_{2}\right), \ldots, w\left(b_{n}\right)\right)^{T}$.

So we can write

$\left(\begin{array}{cc}S_{1} & 0 \\ 0 & S_{2}\end{array}\right)\left(\begin{array}{l}m(x) \\ w(x)\end{array}\right)=\left(\begin{array}{l}m(b) \\ w(b)\end{array}\right)$ 
In order to solve the above linear system we must now calculate $S^{-1}$ (if exists).

Assuming that $\mathrm{S}$ is nonsingular we get

$x=S^{-1} b$

Now $S^{-1}=\left(\begin{array}{cc}S_{1} & 0 \\ 0 & S_{2}\end{array}\right)^{-1}=\left(\begin{array}{cc}S_{1}^{-1} & 0 \\ 0 & S_{2}^{-1}\end{array}\right)$

Therefore the solution is

$x=S^{-1} b$

i.e. $\left(\begin{array}{l}m(x) \\ w(x)\end{array}\right)=\left(\begin{array}{cc}S_{1}^{-1} & 0 \\ 0 & S_{2}^{-1}\end{array}\right)\left(\begin{array}{l}m(b) \\ w(b)\end{array}\right)$

or, $m(x)=S_{1}^{-1} m(b)$ and $w(x)=S_{2}{ }^{-1} w(b)$

Theorem-3.1: (Friedman et al 1998): The matrix $\mathrm{S}$ is non singular if and only if the matrices $S_{1}$ and $S_{2}$ are both nonsingular.

Proof: Since $S=\left(\begin{array}{cc}S_{1} & 0 \\ 0 & S_{1}\end{array}\right)$

Clearly $\operatorname{det}(S)=\operatorname{det}\left(S_{1} S_{2}\right)=\operatorname{det}\left(S_{1}\right) \operatorname{det}\left(S_{2}\right)$

Therefore $\operatorname{det}(S) \neq 0$ if and only if $\operatorname{det}\left(S_{1}\right) \neq 0$ and $\operatorname{det}\left(S_{2}\right) \neq 0$

which concludes the proof.

Theorem-3.2: The necessary and sufficient conditions for the existence of a strong solution:

Let $S=\left(\begin{array}{cc}S_{1} & 0 \\ 0 & S_{2}\end{array}\right)$ be a nonsingular matrix. The system (2) has a strong solution if and only if $S_{2}{ }^{-1} w(b) \geq 0$

Proof: Let us define $m(x)=\left(m\left(x_{1}\right), m\left(x_{2}\right), \ldots, m\left(x_{n}\right)\right)^{T}$,

$w(x)=\left(w\left(x_{1}\right), w\left(x_{2}\right), \ldots, w\left(x_{n}\right)\right)^{T}$

and $m(b)=\left(m\left(b_{1}\right), m\left(b_{2}\right), \ldots, m\left(b_{n}\right)\right)^{T}$,

$w(b)=\left(w\left(b_{1}\right), w\left(b_{2}\right), \ldots, w\left(b_{n}\right)\right)^{T}$

from the system (3.1) we obtain

$\left(\begin{array}{cc}S_{1} & 0 \\ 0 & S_{2}\end{array}\right)\left(\begin{array}{l}m(x) \\ w(x)\end{array}\right)=\left(\begin{array}{l}m(b) \\ w(b)\end{array}\right)$

Hence $S_{1} m(x)=m(b)$ and $S_{2} w(x)=w(b)$

Therefore $w(x)=S_{2}{ }^{-1} w(b)$

If the system (2) has a strong solution then by the definition3, we have

$w(x) \geq 0$

Hence $S_{2}{ }^{-1} w(b) \geq 0$ holds.

Conversely if the above inequality holds then from the relation

$w(x)=S_{2}{ }^{-1} w(b)$ we get $w(x) \geq 0$

Example-3.3: consider the $2 \times 2$ fuzzy linear system

$\widetilde{x_{1}}+\widetilde{x_{2}}=\widetilde{b_{1}}$

$2 \widetilde{x_{1}}+3 \widetilde{x_{2}}=\widetilde{b_{2}}$

where $\widetilde{b_{1}}=(4,5,6,7 ; 0.8), \widetilde{b_{2}}=(11,12,13,14 ; 0.9)$

$\left(\widetilde{b_{1}}\right)_{\propto}=\left[\underline{b_{1}}, \overline{b_{1}}\right]=\left[4+\frac{\alpha}{\omega}, 7-\frac{\alpha}{\omega}\right],\left(\widetilde{b_{2}}\right)_{\propto}=\left[\underline{b_{2}}, \overline{b_{2}}\right]=\left[11+\frac{\alpha}{\omega}, 14-\frac{\alpha}{\omega}\right]$ 
where $\omega=\min (0.8,0.9)=0.8$

$m\left(b_{1}\right)=5.5, w\left(b_{1}\right)=1.5-\frac{\alpha}{\omega}, m\left(b_{2}\right)=12.5, w\left(b_{2}\right)=1.5-\frac{\alpha}{\omega}$

Let us denote the solution $\widetilde{x_{l}}$ as $m\left(x_{i}\right)+K w\left(x_{i}\right), i=1,2$.

So following above we get

$m\left(x_{1}\right)=3 \times 5.5-1 \times 12.5=4$

$m\left(x_{2}\right)=-2 \times 5.5+1 \times 12.5=1.5$

$w\left(x_{1}\right)=3\left(1.5-\frac{\alpha}{\omega}\right)-1\left(1.5-\frac{\alpha}{\omega}\right)=3-\frac{2 \alpha}{\omega}$

$w\left(x_{2}\right)=-2\left(1.5-\frac{\alpha}{\omega}\right)+1\left(1.5-\frac{\alpha}{\omega}\right)=-1.5+\frac{\alpha}{\omega}$

Now as $0<\propto \leq \omega$ so, $w\left(x_{1}\right)>0$ and $w\left(x_{2}\right)<0$

Therefore we get $\widetilde{x_{1}}$ as strong solution and $\widetilde{x_{2}}$ as weak solution and $\widetilde{x_{1}}=(1,3,5,7 ; 0.8), \widetilde{x_{2}}=(0,1,2,3 ; 0.8)$.

\subsection{Fuzzy complex linear system}

Consider the $n \times n$ complex linear system

$$
\begin{aligned}
& c_{11} z_{1}+c_{12} z_{2}+\cdots+c_{1 n} z_{n}=d_{1} \\
& c_{21} z_{1}+c_{22} z_{2}+\cdots+c_{2 n} z_{n}=d_{2} \\
& \vdots \\
& c_{n 1} z_{1}+c_{n 2} z_{2}+\cdots+c_{n n} z_{n}=d_{n}
\end{aligned}
$$

where the co-efficient matrix $C=\left(c_{k j}\right), 1 \leq k \leq n, 1 \leq j \leq \mathrm{n}$, is a crisp complex $n \times n$ matrix and $d=\left(d_{1}, d_{2}, \ldots, d_{n}\right)^{T}$ is a column vector of fuzzy complex numbers and $z_{j}, j=1,2, \ldots, n$ are unknown fuzzy complex numbers.

It means $c_{k j}=a_{k j}+i b_{k j}, z_{j}=x_{j}+i y_{j}$ and $d_{k}=p_{k}+i q_{k}$

Let us now rearrange the linear system so that the unknowns are $m\left(z_{j}\right), w\left(z_{j}\right)$,

$1 \leq j \leq n$, and the right hand side column is

$$
d=\left(m\left(d_{1}\right)+K w\left(d_{1}\right), m\left(d_{2}\right)+K w\left(d_{2}\right), \ldots, m\left(d_{n}\right)+K w\left(d_{n}\right)\right)^{T} .
$$

We get the crisp linear system

$$
\begin{aligned}
& c_{11}\left\{m\left(z_{1}\right),+K w\left(z_{1}\right)\right\}+c_{12}\{ \\
& \left.+\left(z_{2}\right),+K w\left(z_{2}\right)\right\}+\cdots \\
& +c_{1 n}\left\{m\left(z_{n}\right),+K w\left(z_{n}\right)\right\}=\left\{m\left(d_{1}\right),+K w\left(d_{1}\right)\right\} \\
& \vdots \\
& c_{21}\left\{m\left(z_{1}\right),+K w\left(z_{1}\right)\right\}+c_{22}\left\{m\left(z_{2}\right),+K w\left(z_{2}\right)\right\}+\cdots \\
& +c_{2 n}\left\{m\left(z_{n}\right),+K w\left(z_{n}\right)\right\}=\left\{m\left(d_{2}\right),+K w\left(d_{2}\right)\right\} \\
& c_{n 1}\left\{m\left(z_{1}\right),+K w\left(z_{1}\right)\right\}+c_{n 2}\left\{m\left(z_{2}\right),+K w\left(z_{2}\right)\right\}+\cdots \\
& +c_{n n}\left\{m\left(z_{n}\right),+K w\left(z_{n}\right)\right\}=\left\{m\left(d_{n}\right),+K w\left(d_{n}\right)\right\}
\end{aligned}
$$

Comparing both sides we get two linear systems as

$\sum_{j=1}^{n} c_{k j} m\left(z_{j}\right)=m\left(d_{k}\right), k=1,2, \ldots, n$

and $\sum_{j=1}^{n} c_{k j}^{s g n} w\left(z_{j}\right)=w\left(d_{k}\right), k=1,2, \ldots, n$

where $c_{k j}^{s g n}=a_{k j} \operatorname{sgn}\left(a_{k j}\right)+i b_{k j} \operatorname{sgn}\left(b_{k j}\right)$ 
Now solving the above systems we can find $m\left(z_{j}\right)$ and $w\left(z_{j}\right)$.

Further for $j=1,2, \ldots, n$ if $w\left(z_{j}\right) \geq 0$ then we get $z_{j}$ as a strong solution and if $w\left(z_{j}\right)<0$ then we get $z_{j}$ as a weak solution.

Example-3.4: [9]: Consider a $2 \times 2$ fuzzy complex system

$(10-7.5 i) \widetilde{z_{1}}-(6-5 i) \widetilde{z_{2}}=\widetilde{d_{1}}$

$-(6-5 i) \widetilde{z_{1}}+(16+3 i) \widetilde{z_{2}}=\widetilde{d_{2}}$

where $\widetilde{d_{1}}=(4,5,6,7 ; 0.8)+(-1,0,1,2 ; 0.9) i$

$\widetilde{d_{2}}=(-3,-2,-1,0, ; 0.9)+(-4,-3,-2,-1 ; 0.8) i$

$\left(\widetilde{d_{1}}\right)_{\propto}=\left[4+\frac{\alpha}{\omega}, 7-\frac{\alpha}{\omega}\right]+i\left[-1+\frac{\alpha}{\omega}, 2-\frac{\alpha}{\omega}\right],\left(\widetilde{d_{2}}\right)_{\propto}=\left[-3+\frac{\alpha}{\omega},-\frac{\alpha}{\omega}\right]+i\left[-4+\frac{\alpha}{\omega},-1-\frac{\alpha}{\omega}\right]$

where $\omega=\min (0.8,0.9)=0.8$

$m\left(d_{1}\right)=5.5+0.5 i, w\left(d_{1}\right)=\left(1.5-\frac{\alpha}{\omega}\right)+\left(1.5-\frac{\alpha}{\omega}\right) i$,

$m\left(d_{2}\right)=-1.5-2.5 i, w\left(d_{2}\right)=\left(1.5-\frac{\alpha}{\omega}\right)+\left(1.5-\frac{\alpha}{\omega}\right) i$

Let us denote the solution $\widetilde{z_{l}}$ as $m\left(z_{i}\right)+K w\left(z_{i}\right), i=1,2$.

$$
\begin{aligned}
\left(\begin{array}{l}
m\left(z_{1}\right) \\
m\left(z_{2}\right)
\end{array}\right) & =\left(\begin{array}{cc}
10-7.5 i & -6+5 i \\
-6+5 i & 16+3 i
\end{array}\right)^{-1}\left(\begin{array}{c}
5.5+0.5 i \\
-1.5-2.5 i
\end{array}\right)=\left(\begin{array}{r}
-0.0245+0.1731 i \\
0.4631-0.5356 i
\end{array}\right) \\
\left(\begin{array}{l}
w\left(z_{1}\right) \\
w\left(z_{2}\right)
\end{array}\right) & =\left(\begin{array}{cc}
10+7.5 i & 6+5 i \\
6+5 i & 16+3 i
\end{array}\right)^{-1}\left(\begin{array}{l}
\left(1.5-\frac{\alpha}{\omega}\right)+\left(1.5-\frac{\alpha}{\omega}\right) i \\
\left(1.5-\frac{\alpha}{\omega}\right)+\left(1.5-\frac{\alpha}{\omega}\right) i
\end{array}\right) \\
& =\left(\begin{array}{c}
(0.2923+0.0324 i)\left(1.5-\frac{\alpha}{\omega}\right) \\
(0.2520+0.0655 i)\left(1.5-\frac{\alpha}{\omega}\right)
\end{array}\right)
\end{aligned}
$$

Here as $0<\propto \leq \omega, w\left(z_{1}\right)>0$ and $w\left(z_{2}\right)>0$.So we get the strong solutions.

$$
\begin{gathered}
\widetilde{z_{1}}=(-0.4630,-0.1707,0.1217,0.4140 ; 0.8)+i(0.1245,0.1569,0.1893,0.2217 ; 0.8) \\
\widetilde{z_{2}}=(0.0851,0.3371,0.5891,0.8411 ; 0.8)+i(-0.6340,-0.5685,-0.5029,-0.4374 ; 0.8)
\end{gathered}
$$

\section{Conclusion}

Various approaches have been used by the other authors to solve fuzzy real and complex linear equations and linear systems but those are sometimes lengthy or not efficient to compute. In this paper we have used a new approach to solve both real and complex fuzzy linear equations and linear systems. We have also considered numerical examples and solved by using this approach. Further we can also use this approach to fully fuzzy linear systems. There are so many areas in Engineering Sciences where we can handle the problems involving linear systems by this approach.

\section{Acknowledgement}

The reaserch work of Sanhita Banerjee is financed by Department of Science and Technology (No.DST/INSPIRE Fellowship/2012/13), Govt. of India. The authors would like to thank to the Editor and the Reviewer for their constructive comments and suggestions that significantly improve the quality and clarity of the paper. 


\section{References}

[1] Amit Kumar, Neetu, Abhinav Bansal, "A new approach for solving fully fuzzy linear systems", Hindawi Publishing Corporation, Advances in Fuzzy Systems, vol. (2011), Article ID 943161, 8 pages.

[2] Amit Kumar, Neetu, Abhinav Bansal, "A new method to solve fully fuzzy linear system with trapezoidal fuzzy numbers", Canadian Journal on Science and Engineering Mathematics, vol.1 (2010), no. 3.

[3] Didier Dubois, Henri Prade, "Systems of fuzzy linear constraints" (1978).

[4] T. Allahviranloo, N. Mikaeilvand, F. Hoseinzadeh Lotfi, M. Fallah Jelodar, "Fully Fuzzy Linear Systems", International Journal of Applied Operaional Research, vol.1 (2011), no.1, pp.35-48.

[5] J. J. Buckley, Y. Qu, "Solving systems of linear fuzzy equations", Fuzzy Sets and Systems 43 (1991) 33-43.

[6] J. J. Buckley, "Fuzzy input-output analysis", European journal of operational research 39 (1989) 54-60.

[7] J. J. Buckley, Y. Qu, "Solving linear and quadratic fuzzy equations", Fuzzy Sets and Systems 38 (1990) 43-59.

[8] J. J. Buckley, Y. Qu, “On using $\propto$-cuts to evaluate fuzzy equations”, Fuzzy Sets and Systems 38 (1990) 309-312.

[9] J.J. Buckley, Y. Qu, "Solving fuzzy equations: a new solution concept", Fuzzy Sets and Systems 39 (1991) 291-301.

[10] L. A. Zadeh. "Fuzzy sets as a basis for a theory of possibility", Fuzzy Sets and Systems, 1 (1978): 3-28.

[11] L. A. Zadeh, "The concept of a linguistic variable and its application to approximate reasoning", I Information Sciences. 8 (1975), 199-249.

[12] M. Friedman, Ma. Ming, A. Kandel, "Fuzzy linear systems", Fuzzy Sets and Systems 96 (1998) 201-209.

[13] Sahin Emrah Amrahov, Iman N. Askerzade, "Strong Solutions of the Fuzzy Linear Systems".

[14] S. H. Nasseri, M. Gholami, "Linear system of equations with trapezoidal fuzzy numbers", The Journal of Mathematics and Computer Science, vol.3, no.1 (2011) 71-79.

[15] Taher Rafgooy, Hadi Sadghi Yazhi , Reza Monsefi, "Fuzzy Complex System of Linear Equations Applied to Circuit Analysis", International Journal of Computer and Electrical Engineering,vol.1, no.5 (2009), December.

[16] S.H. Nasseri, F. Zahmatkesh, "Huang method for solving fully fuzzy linear system of equations", The Journal of Mathematics and Computer Science, Vol .1 No.1 (2010) 1-5.

[17] Sukanta Nayak, S. Chakraverty, "A New Approach to Solve Fuzzy System of Linear Equations", Journal of Mathematics and Computer Science,vol 7 (2013), 205-212.

[18] S.H.Nasseri, M.Sohrabi, "Gram-Schmidt Approach for Linear System of Equations with Fuzzy Parameters", The Journal of Mathematics and Computer Science, Vol. 1 No.2 (2010) 80-89. 